\title{
A photometric catalogue of southern emission-line stars ${ }^{\star, \star \star}$
}

\author{
D. de Winter ${ }^{1,2,3}$, M. E. van den Ancker ${ }^{4}$, A. Maira ${ }^{1}$, P. S. Thé ${ }^{5}$, H. R. E. Tjin A Djie ${ }^{5}$, I. Redondo ${ }^{1}$, \\ C. Eiroa ${ }^{1}$, and F. J. Molster ${ }^{5,6}$
}

1 Dpto. Física Teórica, C-XI, Universidad Autónoma de Madrid, Cantoblanco, 28049 Madrid, Spain

2 Instituto de Astrofísica de Canarias, C/ Via Láctea s/n, 38200 La Laguna, Tenerife, Spain

3 TNO TPD, Stieltjesweg 1, PO Box 155, 2600 AD Delft, The Netherlands

4 Harvard-Smithsonian Center for Astrophysics, 60 Garden Street, MS 78, Cambridge, MA 02138, USA

5 Astronomical Institute, University of Amsterdam, Kruislaan 403, 1098 SJ Amsterdam, The Netherlands

${ }^{6}$ School of Materials Science and Engineering, Georgia Tech, Atlanta, GA 30332-0245, USA

Received 19 June 2001 / Accepted 18 October 2001

\begin{abstract}
We present a catalogue of previously unpublished optical and infrared photometry for a sample of 162 emission-line objects and shell stars visible from the southern hemisphere. The data were obtained between 1978 and 1997 in the Walraven $(W U L B V)$, Johnson/Cousins $\left(U B V(R I)_{\mathrm{c}}\right)$ and ESO and SAAO near-infrared (JHKLM) photometric systems. Most of the observed objects are Herbig Ae/Be (HAeBe) stars or HAeBe candidates appearing in the list of HAeBe candidates of Thé et al. (1994), although several B[e] stars, LBVs and $\mathrm{T}$ Tauri stars are also included in our sample. For many of the stars the data presented here are the first photo-electric measurements in the literature. The resulting catalogue consists of 1809 photometric measurements. Optical variability was detected in 66 out of the 116 sources that were observed more than once. 15 out of the 50 stars observed multiple times in the infrared showed variability at $2.2 \mu \mathrm{m}$ ( $K$ band).
\end{abstract}

Key words. circumstellar matter - stars: emission-line - stars: pre-main sequence - infrared: stars

\section{Introduction}

One of the classic characteristics of a young star is the presence of photometric variability. Several types of photometric variability can be recognized, amongst which are more or less periodic variability due to magnetic activity on the stellar surface (star spots), brightness variations due to a variable rate of accretion, and irregular largeamplitude $(>0.5)$ variations due to variable circumstellar extinction (Bibo \& Thé 1991; Shevchenko et al. 1993; Herbst et al. 1994; van den Ancker et al. 1998; Herbst \& Shevchenko 1999). However, a detailed analysis of a star's photometric behaviour requires great amounts of data, preferably over a large period of time. In practice, sufficient data can often only be obtained by combining data sets from the literature with new data.

Herbig Ae/Be (HAeBe) stars may be one of the most interesting groups of young stars to study since they

Send offprint requests to: D. de Winter,

e-mail: dolf@xiada.ft.uam.es

* Based on observations collected at the European Southern Observatory, La Silla, Chile and on observations collected at the South African Astronomical Observatory.

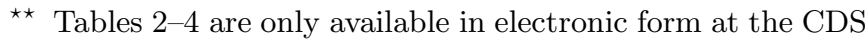
via anonymous ftp to cdsarc.u-strasbg.fr (130.79.128.5) or via

http://cdsweb.u-strasbg.fr/cgi-bin/qcat?J/A+A/380/609 are intrinsically bright and therefore can be seen over great distances, tracing galactic regions of star formation. Furthermore, many are sufficiently bright to be observed with smaller telescopes, allowing detailed studies of their photometric behaviour to be made. A catalogue of 287 candidate members of the Herbig Ae/Be stellar group was published by Thé et al. (1994). For many of the objects in this catalogue little or no data is available and therefore a new investigation of their properties is in order.

In this paper we present photometry of 162 emissionline and shell stars, most of which appear in the list of HAeBe candidates by Thé et al. Names, positions and classifications of the stars are listed in Table 1. Section 2 gives a detailed description of the observations. Although a brief discussion of the data is presented in Sect. 3, we defer a detailed analysis of these data to future papers in which these data can be combined with photometry over a much longer time span.

\section{Observations}

Photometric data in the Walraven $W U L B V$ system of our programme stars were obtained between 1978 and 1988 at the $90 \mathrm{~cm}$ Dutch Light Collector. The data taken in 1978 were obtained when this telescope was located 
Table 1. Properties of programme stars.

\begin{tabular}{|c|c|c|c|c|c|c|c|c|c|c|c|}
\hline \multirow[t]{2}{*}{ Name } & \multirow[t]{2}{*}{ Cat. Des. } & \multirow[t]{2}{*}{ Other } & $\alpha(2000)$ & \multirow[t]{2}{*}{$\delta(2000)$} & \multirow[t]{2}{*}{ Type } & \multicolumn{3}{|c|}{ \# Observations } & \multirow{2}{*}{$\begin{array}{c}V_{\text {obs }} \\
{[\mathrm{mag}]}\end{array}$} & \multirow{2}{*}{$\begin{array}{c}V_{\text {lit }} \\
{[\mathrm{mag}]}\end{array}$} & \multirow[t]{2}{*}{ Ref. } \\
\hline & & & & & & $W U L B V$ & $U B V R I$ & $J H K L M$ & & & \\
\hline 71 Cet & HD 15004 & & 022458.4 & -024648 & A-SII & 2 & - & - & $6.34-6.65$ & $6.31-6.36$ & $(1)$ \\
\hline BU Tau & HD 23862 & MWC 75 & 034911.2 & +240812 & $\mathrm{Be}$ & 1 & - & - & 5.08 & 5.01 & (1) \\
\hline V1080 Tau & HD 283817 & $\mathrm{StH} \alpha 33$ & 044032.6 & +242631 & $\mathrm{HAe}$ & 2 & 3 & - & $10.10-11.68$ & $10.29-10.56$ & $(2)$ \\
\hline \multirow[t]{2}{*}{ AB Aur } & HD 31293 & MWC 93 & 045545.8 & +30 3304 & $\mathrm{HAe}$ & 1 & 2 & - & $7.02-7.04$ & $7.03-7.13$ & (1) \\
\hline & HD 31648 & MWC 480 & 045846.3 & +295037 & $\mathrm{HAe}$ & 2 & 3 & - & $7.68-7.72$ & $7.66-7.86$ & (1) \\
\hline UX Ori & HD 293782 & & 050430.0 & -034714 & HAe & 29 & 13 & 12 & $9.84-11.79$ & $9.66-11.79$ & $(3)$ \\
\hline V1012 Ori & & & 051136.4 & -022247 & $\mathrm{HAe}$ & - & 6 & - & $12.07-13.12$ & $12.5-16.5 \mathrm{pg}$ & $(2)$ \\
\hline S22/LMC & HD 34664 & MWC 105 & 051353.1 & -672654 & $\mathrm{~B}[\mathrm{e}]$ & - & 3 & - & $11.77-12.65$ & & \\
\hline V1366 Ori & HD 34282 & & 051600.5 & -094835 & HAe & - & 3 & - & $9.81-9.94$ & & \\
\hline V346 Ori & HD 287841 & & 052442.8 & +014348 & $\mathrm{HAe}$ & 22 & 6 & 3 & $10.09-10.84$ & $10.17-10.38$ & $(3)$ \\
\hline \multirow[t]{2}{*}{ CO Ori } & $\mathrm{BD}+11809$ & & 052738.3 & +112539 & $\mathrm{HFe}$ & - & 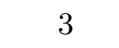 & - & $11.25-11.70$ & $9.81-12.52$ & (4) \\
\hline & HD 35929 & & 052742.8 & -081938 & $\mathrm{HFe}$ & - & 5 & 1 & $8.11-8.31$ & & \\
\hline \multirow[t]{3}{*}{ Par 102} & BD-06 1193 & $\mathrm{StH} \alpha 41$ & 052911.4 & -060805 & $\mathrm{HFe}$ & - & 8 & 3 & $10.43-10.54$ & & \\
\hline & $\mathrm{BD}+11820$ & & 052922.1 & +115050 & HAe? & - & 1 & - & 10.84 & & \\
\hline & HD 36112 & MWC 758 & 053027.5 & +251957 & HAe & - & 2 & - & $8.26-8.27$ & $8.20-8.44$ & (1) \\
\hline \multirow[t]{2}{*}{ HK Ori } & & MWC 497 & 053128.0 & +120911 & $\mathrm{HFe}$ & 23 & 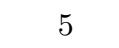 & 3 & $11.41-11.67$ & $11.39-12.10$ & (4) \\
\hline & HD 244604 & & 053157.3 & +111741 & HAe & - & 3 & - & 8.99-9.38 & $9.18-9.71$ & (1) \\
\hline RY Ori & & & 053210.0 & -024949 & $\mathrm{HFe}$ & 8 & 4 & 3 & $11.57-12.68$ & $11.08-12.96$ & $(3)$ \\
\hline EZ Ori & & Par 1409 & 053418.6 & -050448 & $\mathrm{TT}$ & - & 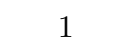 & - & 12.87 & $12.0-14.1 \mathrm{pg}$ & $(2)$ \\
\hline IX Ori & & Par 1552 & 053440.8 & -052243 & $\mathrm{TT}$ & - & 4 & 1 & 12.33 & $13.4-15.5 \mathrm{pg}$ & $(2)$ \\
\hline V372 Ori & HD 36917 & Par 1605 & 053447.0 & -053415 & A-sh & 10 & 3 & 3 & 7.98 & $7.98-8.03$ & (3) \\
\hline YZ Ori & & Par 1648 & 0534 54: & -05 03 30: & $\mathrm{TT}$ & - & 4 & 1 & $13.74-14.85$ & $13.9-16.6 \mathrm{pg}$ & $(2)$ \\
\hline Par 1660 & HD 36939 & & 053455.3 & -053022 & $\mathrm{HBe}$ ? & 9 & 2 & 3 & $8.97-9.00$ & $8.98-9.02$ & $(3)$ \\
\hline Par 1623 & $\mathrm{BD}-051306$ & & 053455.6 & -051643 & A-sh & 10 & 2 & 2 & $10.13-10$ & & \\
\hline KS Ori & & Par 1685 & 053500.1 & -052516 & A-sh & 10 & 2 & 1 & $10.05-10.12$ & $9.7-12.0 \mathrm{pg}$ & $(2)$ \\
\hline V1271 Ori & HD 245185 & & 053509.6 & +100152 & HAe & - & 6 & - & $9.91-9.98$ & $9.85-9.95$ & $(3)$ \\
\hline MR Ori & & & 053517.0 & -052146 & A-sh & - & 3 & - & $9.54-10.85$ & $10.3-12.0$ & $(2)$ \\
\hline LZ Ori & HD 294263 & Par 1854 & 053517.8 & -044107 & A-sh & 20 & 1 & 3 & $9.73-10.09$ & $10.3-11.1 \mathrm{pg}$ & (2) \\
\hline MX Ori & BD-05 1317 & Par 1953 & 05352 & -050916 & $\mathrm{HFe}$ & - & 1 & - & & $1.7 \mathrm{pg}$ & $(2)$ \\
\hline NV Ori & $\mathrm{BD}-051324$ & Par 2086 & 053531.3 & -053309 & $\mathrm{HFe}$ & 6 & - & 2 & $9.63-9.90$ & $9.78-10.35$ & $(3)$ \\
\hline V361 Ori & HD 37062 & Par 2085 & 053531.4 & -052516 & B-sh & 9 & 1 & 3 & 8.15 & $8.14-8.24$ & (3) \\
\hline T Ori & BD-05 1329 & MWC 763 & 053550.4 & -052835 & $\mathrm{HAe}$ & 25 & 10 & 7 & $10.15-11.04$ & $10.00-11.95$ & $(3)$ \\
\hline CQ Tau & HD 36910 & & 053558.5 & +244454 & $\mathrm{HFe}$ & 1 & 4 & - & $10.22-11.06$ & $9.36-11.14$ & (3) \\
\hline V380 Ori & $\mathrm{BD}-061253$ & MWC 765 & 053625.4 & -064258 & $\mathrm{HAe}$ & 25 & 12 & 7 & $10.21-$ & 11.22 & (4) \\
\hline BN Ori & HD 245465 & & 053629.3 & +065002 & A-sh & 11 & 3 & 2 & $9.62-9.68$ & $9.56-9.71$ & (5) \\
\hline Par 2441 & BD-04 1191 & & 053651.2 & -042540 & $\mathrm{TT}$ & - & 1 & - & 10.74 & & \\
\hline V586 Ori & HD 37258 & Par 2473 & 053659.3 & -060916 & $\mathrm{HAe}$ & 24 & 8 & 3 & $9.64-10.08$ & 0.77 & $(3)$ \\
\hline BF Ori & BD-06 1259 & & 05371 & -063501 & $\mathrm{HAe}$ & 25 & 14 & 5 & 9.8 & 11.92 & $(3)$ \\
\hline Par 2599 & HD 37357 & KMS 27 & 053747.1 & -064230 & $\mathrm{HAe}$ & - & 7 & 2 & $8.83-8.84$ & $8.83-8.91$ & (4) \\
\hline N3Sk81 & & KMS 34 & 053808.8 & -064913 & HAe & - & 6 & 1 & $13.75-13.84$ & & \\
\hline Par 2653 & HD 3 & & 053814.5 & -052513 & $\mathrm{HAe}$ & 2 & 10 & 1 & $9.76-9.85$ & $9.79-9.81$ & $(4)$ \\
\hline & CD-59 1105 & & 053833.0 & -590428 & $\mathrm{Fe}$ & - & 1 & - & 9.74 & & \\
\hline N3Sk83 & $=$ V883 Ori? & KMS 40 & 053818.1 & -070226 & $\mathrm{HBe}$ & - & 1 & 1 & 14.4 & & \\
\hline V599 Ori & & KMS 49 & 053854.4 & -071638 & HAe & - & 2 & 1 & $13.76-13.80$ & $16.3 \mathrm{pg}$ & $(2)$ \\
\hline$\omega$ Ori & HD 37490 & MWC 117 & 053911.1 & +040717 & $\mathrm{Be}$ & - & 3 & - & $4.48-4.56$ & $4.49-4.55$ & (1) \\
\hline V350 Ori & & & 054011.6 & $-0942 \quad 10$ & HAe & 4 & 16 & 3 & $10.77-13.32$ & $10.71-13.24$ & (3) \\
\hline KMS 82 & & & 054037.2 & -08 $0402:$ & $\mathrm{HFe}$ ? & - & 4 & 2 & $11.55-14.57$ & & \\
\hline KMS 108 & & & 054046.3 & -08 53 48: & $\mathrm{HBe} ?$ & - & 2 & 1 & $11.19-11.21$ & & \\
\hline & HD 37806 & MWC 120 & 054102.3 & -024301 & HAe & 9 & 7 & 2 & $7.86-7.96$ & $7.89-7.98$ & (3) \\
\hline KMS & & & 054103.9 & -09 23 18: & $\mathrm{HFe}$ & - & 3 & 1 & $14.80-14.92$ & & \\
\hline V351 Ori & HD 38238 & & 054418.8 & +000840 & HAe & 8 & 2 & 4 & $8.89-9.13$ & 10.94 & $(6)$ \\
\hline FU Ori & $\mathrm{BD}+095427$ & CDS 535 & 054522.4 & +090412 & FUOR & 2 & 1 & - & $9.09-9.32$ & $8.02-10.06$ & (4) \\
\hline & HD 288313 & & 055403.0 & +014022 & $\mathrm{TT}$ & - & 7 & - & $9.45-9.95$ & & \\
\hline V1307 Ori & HD 250550 & MWC 789 & 060200.0 & +163057 & $\mathrm{HBe}$ & 14 & - & 2 & $9.53-9.56$ & $9.54-9.88$ & $(3)$ \\
\hline 17 Lep & HD 41511 & MWC 519 & 060459.1 & -162904 & A-sh & 2 & 3 & - & $4.91-5.01$ & $4.97-5.02$ & (1) \\
\hline $\mathrm{LkH} \alpha 208$ & & & $\begin{array}{lll}06 & 07 & 49.4\end{array}$ & +183927 & HAe & - & 3 & - & $11.04-11.50$ & $11.36-12.21$ & (4) \\
\hline $\mathrm{LkH} \alpha 339$ & & & 061054.4 & -061439 & HAe & - & 5 & 1 & $13.44-13.64$ & $13.65-13.67$ & (4) \\
\hline
\end{tabular}


Table 1. continued.

\begin{tabular}{|c|c|c|c|c|c|c|c|c|c|c|c|}
\hline \multirow[t]{2}{*}{ Name } & \multirow[t]{2}{*}{ Cat. Des. } & \multirow[t]{2}{*}{ Other } & $\alpha(2000)$ & $\delta(2000)$ & \multirow[t]{2}{*}{ Type } & \multicolumn{3}{|c|}{ \# Observations } & \multirow{2}{*}{$\begin{array}{c}V_{\text {obs }} \\
{[\mathrm{mag}]}\end{array}$} & \multirow{2}{*}{$\begin{array}{c}V_{\text {lit }} \\
{[\mathrm{mag}]}\end{array}$} & \multirow[t]{2}{*}{ Ref. } \\
\hline & & & $\mathrm{h}$ & & & $\overline{W U L B V}$ & $\overline{U B V}$ & $J H K L M$ & & & \\
\hline V1308 Ori & & MWC 137 & 061845.5 & +151652 & $\mathrm{HBe}$ & - & 2 & - & $11.67-11.98$ & $11.79-12.27$ & $(4)$ \\
\hline FS CMa & HD 45677 & MWC 142 & 062817.4 & -130311 & $\mathrm{~B}[\mathrm{e}]$ & 17 & 13 & 2 & $7.94-8.74$ & $7.22-8.85$ & (7) \\
\hline VY Mon & & & 063106.9 & +102605 & $\mathrm{HBe}$ & - & 3 & - & 12.97-13.09 & $12.77-14.77$ & $(4)$ \\
\hline V699 Mon & & $\mathrm{LkH} \alpha 215$ & 063241.8 & +100934 & $\mathrm{HBe}$ & 19 & 5 & 2 & $10.46-10.91$ & $10.36-10.84$ & $(2)$ \\
\hline V700 Mon & HD 259431 & MWC 147 & 063305.2 & +101920 & $\mathrm{HBe}$ & 13 & 6 & 2 & $8.69-8.75$ & $8.65-8.97$ & (1) \\
\hline R Mon & $\mathrm{BD}+081427$ & MWC 151 & 063909.9 & +084410 & $\mathrm{HBe}$ & 13 & 4 & 2 & $10.35-12.41$ & $11.60-12.54$ & $(4)$ \\
\hline V590 Mon & W90 & $\mathrm{LkH} \alpha 25$ & 064044.6 & +094802 & $\mathrm{HFe}$ & - & 8 & 1 & $11.88-12.94$ & $12.60-12.95$ & $(4)$ \\
\hline ST Pup & CD-37 3101 & & 064856.4 & -371633 & Ceph. & - & 1 & - & 10.31 & $9.28-10.68$ & $(2)$ \\
\hline V743 Mon & HD 50138 & MWC 158 & 065133.4 & -065759 & $\mathrm{~B}[\mathrm{e}]$ & 20 & 13 & 2 & $6.59-6.85$ & $6.53-6.65$ & (1) \\
\hline GU CMa & HD 52721 & MWC 164 & 070149.5 & -111803 & $\mathrm{HBe}$ & 1 & 2 & - & $6.50-6.57$ & $6.52-6.71$ & (1) \\
\hline $\mathrm{Z}$ CMa & HD 53179 & MWC 165 & 070343.2 & -113306 & FUOR & 19 & 19 & 6 & $8.68-10.27$ & $8.61-10.22$ & (4) \\
\hline $\mathrm{HU} \mathrm{CMa}$ & & $\mathrm{LkH} \alpha 220$ & 070406.8 & -112607 & $\mathrm{HBe}$ & - & 3 & - & $11.52-11.92$ & $11.57-12.19$ & $(4)$ \\
\hline V750 Mon & HD 53367 & MWC 166 & 070425.5 & -102716 & $\mathrm{HBe}$ & 12 & 4 & 3 & $6.92-7.08$ & $7.00-7.22$ & $(1)$ \\
\hline NX Pup & CD-44 3318 & Hen 3-32 & $07 \quad 1928.3$ & -443511 & HAe & 15 & 7 & 3 & $9.63-11.08$ & $9.53-10.99$ & (3) \\
\hline \multirow[t]{4}{*}{ V694 Mon } & & MWC 560 & 072551.3 & -074408 & Symb. & - & 8 & - & $9.96-10.33$ & $9.10-10.10$ & (2) \\
\hline & HD 58647 & & 072556.1 & -141044 & A-sh & - & - & 1 & & $6.76-6.88$ & (1) \\
\hline & HD 59319 & MWC 843 & 072836.8 & -215749 & $\mathrm{HBe} ?$ & - & 4 & - & 8.29 & $8.19-8.47$ & (1) \\
\hline & HD 59771 & & 073051.1 & -181543 & $\mathrm{Fe}$ & - & 2 & - & 8.89 & $8.83-9.31$ & (1) \\
\hline Hen 3-40 & & Wray 15 & 0731 48: & $-413400:$ & $\mathrm{Ge}$ & - & 3 & 1 & $13.73-14.42$ & & \\
\hline PW Pup & CD-30 5135 & Hen 3-83 & 074906.0 & -310743 & $\mathrm{Fe}$ & - & 2 & - & $9.23-9.24$ & 9.97 & (1) \\
\hline V402 Pup & HD 64315 & & 075220.3 & -262547 & $\mathrm{Oe}$ & - & 1 & - & 9.24 & 8.99-9.39 & (1) \\
\hline AS 202 & CD-37 4833 & Hen 3-174 & 083235.8 & -375902 & HBe? & - & 1 & - & 10.80 & $9.70-10.70$ & (2) \\
\hline ESO $313-10$ & & & $0842 \quad 17.0$ & $-404413:$ & $\mathrm{HBe}$ & - & 3 & 1 & $14.24-14.59$ & & \\
\hline Hen 3-209 & & Wra $15-285$ & $0848 \quad 45.5$ & -460508 & $\mathrm{~B}[\mathrm{e}]$ & - & 1 & - & 13.56 & & \\
\hline Hen $2-14$ & & Wra $16-30$ & 085159.5 & $-461805:$ & $\mathrm{B}[\mathrm{e}]$ & - & 1 & - & 14.05 & & \\
\hline OU Vel & HD 76534 & Hen 3-225 & 085508.7 & -432800 & $\mathrm{HBe}$ & 2 & 2 & 2 & 7.96 & & \\
\hline RCW 34 & & Gum 19 & 085627.8 & $-430546:$ & $\mathrm{O}$ & - & 2 & - & $11.21-1$ & & \\
\hline Herbst 28 & & Bran 215 & 085826.3 & -432610 & $\mathrm{Be}$ & - & 2 & 1 & $11.25-14.76$ & & \\
\hline \multirow[t]{4}{*}{ RCW 36} & & Bran 217 & 085900.9 & -434410 & $\mathrm{HFe}$ ? & - & 1 & - & 15.20 & & \\
\hline & HD 309784 & Hen $3-315$ & 094236.4 & -670854 & $\mathrm{~B}[\mathrm{e}]$ & - & 1 & - & 10.16 & & \\
\hline & HD 85567 & Hen & 095028.5 & -605803 & $\mathrm{HBe}$ & - & 33 & - & 8.49 & & (1) \\
\hline & HD 87643 & MWC 198 & 100430.3 & -583952 & $\mathrm{~B}[\mathrm{e}]$ & 22 & 16 & 3 & $8.52-9.00$ & $8.68-9.20$ & (3) \\
\hline \multirow[t]{2}{*}{17 Sex } & HD 88195 & & 101007.5 & -082429 & B-sh & 1 & - & - & 5.90 & $5.87-5.93$ & (1) \\
\hline & HD 89249 & 0 & 101620.6 & -553551 & $\mathrm{Be}$ & - & 1 & 1 & 8.90 & $8.70-9.15$ & (1) \\
\hline HR Car & HD 90177 & MWC 202 & 102253.8 & -593728 & $\mathrm{LBV}$ & 21 & 11 & 3 & $7.44-8.42$ & $7.40-8.34$ & (3) \\
\hline Hen 3-416 & & Th $35-40$ & 102544.5 & -583352 & HBe? & - & 1 & - & 10.59 & & \\
\hline Wra $15-566$ & & & 102551.7 & -605314 & $\mathrm{TT} ?$ & - & 2 & - & $13.87-13.90$ & & \\
\hline \multirow[t]{5}{*}{ V503 Car } & HD 90578 & Hen 3-418 & 102600.2 & -574937 & $\mathrm{~B}[\mathrm{e}]$ & - & 1 & - & 9.33 & $9.08-9.75$ & (1) \\
\hline & HD 92061 & Hen 3-455 & 103624.2 & -585709 & HBe? & - & 1 & - & 8.97 & $8.99-9.87$ & (1) \\
\hline & CPD-59 2617 & Hen 3-480 & 104449.4 & -59 49 28: & HBe? & - & - & 1 & & & \\
\hline & HD 94509 & Hen 3-515 & 105327.3 & -582525 & $\mathrm{HBe}$ & - & 4 & - & $9.08-9.13$ & $8.85-9.41$ & (1) \\
\hline & CPD-59 2 & Wra 15-689 & 105555.4 & -601420 & $\mathrm{~B}[\mathrm{e}]$ & - & 1 & - & 10.54 & & \\
\hline GG Car & HD 94878 & MWC 215 & 105558.9 & -602333 & $\mathrm{~B}[\mathrm{e}]$ & 23 & 14 & 3 & $8.53-9.03$ & 0 & (8) \\
\hline & HD 305773 & CD-59 3426 & 105603.9 & -602938 & HBe? & - & 1 & - & 9.08 & 8.80-9.39 & (1) \\
\hline AG Car & HD 94910 & MWC 216 & 105611.6 & -602713 & LBV & 22 & 11 & 3 & $6.49-7.00$ & $6.10-8.12$ & (9) \\
\hline HR 4329 & HD 96706 & & 110649.9 & -705241 & B-sh & 2 & - & - & $5.58-5.59$ & $5.54-5.60$ & (1) \\
\hline DI Cha & $\mathrm{CD}-76486$ & Hen 3 & 110720.7 & -773807 & $\mathrm{TT}$ & 23 & 3 & 3 & $10.65-10.74$ & $10.66-10.74$ & $(10)$ \\
\hline CU Cha & HD 97048 & Hen 3-597 & 110803.3 & -773917 & HAe & 23 & 9 & 7 & $8.40-8.48$ & $8.44-8.48$ & $(10)$ \\
\hline & HD 97240 & & 110918.1 & -774740 & F-sh & 2 & - & - & $8.49-8.50$ & $8.42-8.69$ & (1) \\
\hline & HD 97300 & & 110950.0 & -763648 & B-sh & - & 4 & 1 & $9.02-9.08$ & $8.97-9.03$ & (1) \\
\hline & HD 98922 & Hen 3-644 & 112231.7 & -532211 & $\mathrm{HBe}$ & 2 & 38 & - & $6.72-6.81$ & $6.72-6.83$ & (1) \\
\hline KR Mus & HD 100546 & Hen 3-672 & 113325.4 & -701141 & $\mathrm{HBe}$ & 2 & 4 & 1 & $6.69-6.74$ & $6.71-6.77$ & (3) \\
\hline & HD 101412 & Hen 3-692 & 113944.5 & -601028 & $\mathrm{HBe}$ & - & 6 & 1 & $9.24-9.28$ & $8.97-9.56$ & (1) \\
\hline V644 Cen & HD 306989 & Hen 3-700 & 114306.5 & -604405 & $\mathrm{Be}$ & - & 1 & 1 & 10.52 & $9.91-10.33$ & (10) \\
\hline DX Cha & HD 104237 & Hen 3-741 & 120005.1 & $-78 \quad 1135$ & HAe & 2 & 38 & 1 & $6.49-6.59$ & $6.56-6.68$ & (1) \\
\hline & HD 105234 & & 120705.5 & -784428 & A-sh & 2 & - & - & $7.48-7.48$ & $7.45-7.59$ & (1) \\
\hline RU Cen & HD 105578 & Hen 3-755 & 120923.8 & -452535 & $\mathrm{Ge}$ & - & 1 & - & 8.63 & $8.78-9.56$ & (1) \\
\hline
\end{tabular}


Table 1. continued.

\begin{tabular}{|c|c|c|c|c|c|c|c|c|c|c|c|}
\hline Name & Cat. Des. & Other & $\alpha(2000)$ & $\delta(2000)$ & Type & $\#($ & Observat & ions & $V_{\text {obs }}$ & $V_{\text {lit }}$ & Ref. \\
\hline & & & h $\mathrm{m}$ & 0,11 & & $\overline{W U L B V}$ & $U B V R I$ & $J H K L M$ & g] & {$[\mathrm{mag}]$} & \\
\hline Hen 3-759 & & & 121208.6 & -622901 & $\mathrm{Be}$ & - & 1 & - & 10.47 & & \\
\hline DK Cha & IRAS12496-7650 & & 125316.1 & -77 $0702:$ & : HAe & - & - & 1 & & & \\
\hline V1028 Cen & CD-48 7859 & Hen $3-847$ & 130117.8 & -485319 & HAe & - & 34 & 2 & $10.56-10.65$ & & \\
\hline LSS 3027B & CPD-61 3587B & & 131904.0 & -623410 & $\mathrm{HBe}$ & 1 & 2 & 3 & $15.17-15.24$ & & \\
\hline CQ Cir & HD 130437 & Hen 3-1031 & 145050.3 & $-6017 \quad 10$ & $\mathrm{HBe}$ & - & 7 & 1 & $9.85-9.91$ & & \\
\hline SS73 44 & & & 150323.9 & -632254 & $\mathrm{HBe}$ & - & 2 & 1 & $14.75-16.80$ & & \\
\hline & HD 132947 & & 150456.1 & -630753 & HAe & - & 1 & - & 8.91 & $8.73-9.12$ & $(1)$ \\
\hline HT Lup & CD-33 10685 & Hen 3-1095 & 154512.9 & -341731 & $\mathrm{TT}$ & 9 & - & - & $10.21-10.26$ & $10.26-10.40$ & $0(2)$ \\
\hline & HD 140817 & & 154704.5 & -353037 & B-sh & 2 & - & - & $6.83-6.84$ & $6.77-6.90$ & (1) \\
\hline & HD 141569 & & 154957.7 & -035516 & HAe & - & 1 & - & 7.10 & $7.11-7.14$ & $(3)$ \\
\hline & HD 144432 & Hen 3-1141 & 160658.0 & -274310 & HAe & - & 1 & - & 8.17 & $8.08-8.35$ & (1) \\
\hline V856 Sco & HD 144668 & HR 5999 & 160834.3 & -390618 & HAe & 9 & 26 & 13 & $6.55-7.32$ & $6.64-7.95$ & (3) \\
\hline V1027 Sco & HD 144667 & HR 6000 & 160834.6 & -390534 & B-sh & 7 & 8 & 9 & $6.65-6.73$ & $6.65-6.67$ & (3) \\
\hline & HD 147196 & & 162119.2 & -234229 & $\mathrm{Be}$ & - & 5 & 2 & $7.00-7.04$ & $7.02-7.12$ & (1) \\
\hline Wra $15-1484$ & & Hen 3-1191 & 162714.2 & -483928 & $\mathrm{~B}[\mathrm{e}]$ & - & 44 & - & $12.52-13.91$ & & \\
\hline V2307 Oph & HD 150193 & MWC 863 & 164017.9 & -235345 & HAe & 3 & 11 & 1 & $8.80-8.88$ & $8.79-8.87$ & $(10)$ \\
\hline AK Sco & HD 152404 & & 165444.8 & -365319 & $\mathrm{HFe}$ & 3 & 5 & 2 & $8.81-9.18$ & $8.76-9.93$ & (3) \\
\hline AS 215 & HD 322422 & Hen 3-1293 & 165723.9 & -402140 & $\mathrm{~B}[\mathrm{e}]$ & - & 6 & - & $9.68-9.71$ & $9.71-9.75$ & $(3)$ \\
\hline V921 Sco & CD-42 11721 & MWC 865 & 165906.9 & -424208 & $\mathrm{~B}[\mathrm{e}]$ & 4 & 7 & 4 & $11.10-11.36$ & $11.43-11.73$ & $3(2)$ \\
\hline V1104 Sco & HD 326823 & Hen 3-1330 & 170653.9 & -423640 & $\mathrm{~B}[\mathrm{e}]$ & - & 8 & - & 8.98-9.04 & $8.90-9.33$ & (1) \\
\hline KK Oph & & Hen 3-1346 & 171008.1 & -271518 & $\mathrm{HAe}$ & 3 & 7 & 1 & $11.32-12.36$ & $11.37-11.87$ & $7(3)$ \\
\hline Wra $15-1651$ & & & 171445.6 & $-361835:$ & : HBe? & - & - & 1 & & & \\
\hline Wra $15-1678$ & & & 172014.8 & $-373935:$ & HBe? & - & - & 1 & & & \\
\hline & HD 156702 & MWC 257 & 172050.6 & -383909 & $\mathrm{~B}[\mathrm{e}]$ & - & 4 & - & $8.70-8.72$ & $8.58-8.83$ & (1) \\
\hline $51 \mathrm{Oph}$ & HD 158643 & HR 6519 & 173125.0 & -235746 & $\mathrm{Be}$ & 2 & 7 & - & $4.77-4.81$ & $4.77-4.82$ & (1) \\
\hline XX Oph & HD 161114 & MWC 269 & 174356.5 & -061609 & Symb. & 11 & 5 & 1 & $8.80-9.08$ & $8.80-9.11$ & (3) \\
\hline & HD 163296 & MWC 275 & 175621.3 & -215722 & $\mathrm{HAe}$ & 8 & 15 & 4 & $6.82-6.93$ & $6.82-6.93$ & (1) \\
\hline $\mathrm{LkH} \alpha 108$ & & & 180350.6 & -242111 & HBe? & 2 & - & - & $11.67-11.71$ & & \\
\hline V4203 Sgr & CD-24 13830 & $\mathrm{LkH} \alpha 112$ & 180422.5 & -242210 & $\mathrm{HBe}$ & 2 & 2 & 1 & $9.95-9.98$ & $9.54-10.19$ & (4) \\
\hline & HD 164906 & MWC 280 & 180425.8 & -242308 & Oe & 3 & 2 & - & $7.46-7.49$ & $7.32-7.59$ & (1) \\
\hline $\mathrm{LkH} \alpha 115$ & & & 180450.4 & -242542 & $\mathrm{HBe} ?$ & 3 & 2 & 1 & $11.95-11.96$ & $10.80-12.25$ & $5(4)$ \\
\hline $\mathrm{LkH} \alpha 118$ & CD-24 13874 & Hen 3-1583 & 180549.6 & -241520 & $\mathrm{Be}$ & 5 & 6 & 3 & $11.11-11.18$ & $10.69-11.61$ & $1(4)$ \\
\hline $\mathrm{LkH} \alpha 119$ & & & $180556:$ & -241424 : & : Be & 5 & 6 & 3 & $12.15-12.21$ & $11.27-12.76$ & $6(4)$ \\
\hline NZ Ser & & MWC 297 & 182739.6 & -034952 & $\mathrm{HBe}$ & 6 & 5 & - & $12.03-12.63$ & $11.95-12.60$ & $(4)$ \\
\hline VV Ser & & & 182847.9 & +000840 & HAe & 1 & 4 & - & $11.81-12.31$ & $11.18-13.09$ & $9(4)$ \\
\hline V431 Sct & & MWC 300 & 182925.7 & -060437 & $\mathrm{~B}[\mathrm{e}]$ & 1 & 1 & - & $11.73-11.79$ & $11.54-11.90$ & $(4)$ \\
\hline AS 310 & & $\mathrm{MH} \alpha 375-17$ & 183321.2 & -045807 & $\mathrm{HBe}$ & 1 & 1 & 4 & $12.47-12.59$ & $12.08-13.19$ & $9(4)$ \\
\hline HR 7169 & HD 176269 & & 190103.2 & -370339 & B-sh & 2 & - & - & $6.72-6.74$ & $6.63-6.73$ & (1) \\
\hline HR 7170 & HD 176270 & & 190104.3 & -370342 & B-sh & 1 & - & - & 6.45 & $6.34-6.44$ & (1) \\
\hline $\mathrm{S} \mathrm{CrA}$ & & Hen 3-1731 & 1901 20: & -3657 00: & : TT & 8 & - & - & $10.46-11.76$ & $10.80-12.44$ & $4(4)$ \\
\hline & HD 176386 & & 190138.9 & -365327 & B-sh & 11 & 6 & 3 & $7.27-7.43$ & $7.27-7.30$ & (3) \\
\hline TY CrA & CD-37 13024 & & 190140.8 & -365234 & A-sh & 12 & 6 & 5 & $9.34-9.70$ & $9.06-9.79$ & (3) \\
\hline $\mathrm{R}$ CrA & CD-37 13027 & Hen 3-1733 & 190153.7 & -365708 & HAe & 8 & 10 & 6 & $12.10-13.72$ & $10.18-14.65$ & $5(4)$ \\
\hline $\mathrm{T}$ CrA & & & 190158.8 & -365749 & $\mathrm{HFe}$ & 7 & 2 & 4 & $13.27-14.10$ & 12.10-13.99 & $(4)$ \\
\hline & HD 179218 & MWC 614 & 191111.3 & +154716 & $\mathrm{HBe}$ & 1 & - & - & 7.39 & $7.34-7.47$ & (1) \\
\hline WW Vul & HD 344361 & MWC 987 & 192558.8 & +211231 & HAe & 10 & 8 & - & $10.48-11.68$ & $10.25-12.40$ & $(4)$ \\
\hline V1295 Aql & HD 190073 & MWC 325 & 200302.5 & +054417 & HAe & 1 & - & - & 7.86 & $7.84-7.89$ & (10) \\
\hline HR 7836 & HD 195325 & MWC 1019 & 203018.0 & +105345 & HBe? & 2 & - & - & $5.97-6.03$ & $6.00-6.09$ & (1) \\
\hline Total: & 162 stars & & & & & 753 & 830 & 229 & & & \\
\hline
\end{tabular}

Explanation of the abbreviations used: HAe/Be = Herbig Ae/Be star; HFe = F-type Herbig-like star; TT = T Tauri star; FUOR = FU Orionis type star; A-, B-sh: A- or B- type star with shell lines; Be = classical Be star; B $[\mathrm{e}]=\mathrm{B}[\mathrm{e}]$ star; Symb. = symbiotic star; Ceph. $=$ Cepheid; LBV $=$ Luminous Blue Variable.

References: (1) Tycho Catalogue (ESA 1997); (2) General Catalogue of Variable Stars (Kholopov et al. 1998); (3) ESO Long Term Photometry of Variables data (Manfroid et al. 1991, 1995; Sterken et al. 1993, 1995); (4) Herbst \& Shevchenko (1999); (5) Shevchenko et al. (1997); (6) van den Ancker et al. (1996); (7) de Winter \& van den Ancker (1997); (8) Gosset et al. (1984); (9) Sterken et al. (1996); (10) Kilkenny et al. (1985). 
in Hartebeespoortdam, South Africa. All observations in later years were obtained after the telescope had been moved to the European Southern Observatory, La Silla, Chile. A detailed description of the Walraven photometric system can be found in de Geus et al. (1989), who also explain the employed measuring and data reduction procedures. All data presented here were made with a 5-channel photometer which measures all Walraven $W U L B V$ intensities simultaneously, thereby avoiding any systematic effects due to non-simultaneous measurements at the different photometric passbands. A circular diaphragm with a diameter of $21^{\prime \prime} .5$ was used for all stars. Typical errors in the data, given in $\log _{10}$ intensity values, are $0.004,0.003$, $0.004,0.006$, and 0.009 for $V, V-B, B-U, U-W$, and $B-L$, respectively. Results of the photometric measurements in the Walraven system are listed in Table 2 (only available electronically at the CDS). The last column in this table lists the corresponding $V$ magnitude in the Johnson photometric system, computed using the transformation formula by Brand \& Wouterloot (1988). Also listed in Table 2 are the date and heliocentric Julian date (JD) of each observation. In some cases, the exact universal time of individual observations proved impossible to reconstruct. In those cases, only the integer fraction of the JD is listed.

Optical photometry in the Johnson/Cousins $U B V(R I)_{\text {c }}$ system was obtained at La Silla with the ESO $50 \mathrm{~cm}$ and $1 \mathrm{~m}$ telescopes, during several observing runs between 1979 and 1994. Additional $U B V(R I)_{\mathrm{c}}$ photometry was obtained in February 1997 at the South African Astronomical Observatory (SAAO) using the SAAO $75 \mathrm{~cm}$ telescope. During these observations, mostly made through a $15^{\prime \prime}$ circular diaphragm, the telescope was equipped with a single channel photometer and either a Quantacon RCA 3103A (pre-1993) or EMI 9658RA (post1993) photo-multiplier. The observations through $U, B$, $V, R_{\mathrm{c}}$ and $I_{\mathrm{c}}$ filters were made consecutively, in ascending or in descending order. Data were reduced with the Dutch method, using the E-region standard stars from the list by Graham (1982). Non-variable K and M0 standards were always included to avoid large transformation errors. Typical errors in the resulting data, listed in Table 3 (only available electronically at the CDS), are $0 .{ }^{\mathrm{m}} 01$, 0. 01, 0. $02,0 .{ }^{\mathrm{m}} 01,0{ }^{\mathrm{m}} 02$ for $V, B-V, U-B, V-R$ and $V-I$, respectively. For stars fainter than 12 th magnitude the errors may be twice those values. A colon following the data indicates that these particular data are more uncertain than the values listed here. Two colons indicates very uncertain data.

Near-IR photometric data in the ESO JHKLM system (Bouchet et al. 1989, 1991), of the programme stars were obtained with the ESO $1 \mathrm{~m}$ telescope at La Silla on several occasions between 1979 and 1995. Additional Near-IR observations in the SAAO JHKLM system (Carter 1990; Carter \& Meadows 1995) were obtained with the $1.9 \mathrm{~m}$ telescope at the SAAO in 1997. During the near-IR observations, made through a $13^{\prime \prime}$ diaphragm, the telescope was equipped with a photovoltaic $\mathrm{InSb}$ detector unit. Standard stars were obtained from the list by Bouchet et al. (1991). Sky subtraction was achieved by chopping, with a frequency of $8 \mathrm{~Hz}$, in the east-west direction with a throw of $30^{\prime \prime}$ amplitude. The $J, H, K, L$ and $M$ magnitudes were again measured consecutively, in ascending or in descending order. Typical errors in these data are $0 .{ }^{\mathrm{m}} 04,0 .{ }^{\mathrm{m}} 04,00^{\mathrm{m}} 03,0{ }^{\mathrm{m}} 05,0{ }^{\mathrm{m}} 06$ for the $J, H, K$, $L$ and $M$ magnitudes, respectively. The resulting near-IR magnitudes of our programme stars are listed in Table 4 (only available electronically at the CDS).

\section{Discussion}

A summary of all 1809 new photometric measurements is given in the last four columns of Table 1, which list the number of obtained measurements in the Walraven, Johnson/Cousins and Near-IR photometric systems, as well as the observed range in $V$ magnitude. Note that for some stars with a small range in $V$, this is entirely compatible with the expected errors in our measurements. Also listed in Table 1 is the range of photometric values found in the literature. Ranges derived from photographic rather than photo-electric data are indicated by the suffix "pg". In most cases the range observed by us is within the previously known range. In other cases (e.g. V1080 Tau), the data presented here expands the known variability range. In some objects (e.g. $\mathrm{LkH} \alpha 339, \mathrm{~V} 921 \mathrm{Sco}$ ) the range in $V$ magnitude observed by us is completely out of the range known from literature. In those cases one might expect the star to show variations on time scales longer than those covered by our data.

Although a detailed analysis of all the data presented here is beyond the scope of this paper, we note that the statistical properties of the observed variations are in good agreement with previous work. Of the 116 stars with more than one measurement in Tables 2 or 3, 66 (or 57\%) show variability in the $V$ band with a magnitude exceeding 0.1 . This is very close to the $65 \%$ of HAeBes showing significant variations in the study by van den Ancker et al. (1998). The slightly lower fraction found here can easily be explained by the smaller number of measurements per star in the current study, causing us to not detect variability in some known variables.

In the case of near-infrared variability, literature data is much more scarce (in fact, for many of the stars the data listed in Table 4 are the first JHKLM measurements in the literature). Therefore a comparison with previous work is more cumbersome. Of the 50 stars with more than one measurement in Table 4,15 show significant $(>0.2)$ variability in their $K(2.2 \mu \mathrm{m})$ magnitude. Again we must caution that in view of the limited amount of measurements per star this will certainly be an underestimate of the number of near-infrared variables in our sample. In fact, in some cases the magnitudes we provide differ by more than 0.5 with determinations from the literature (Gezari et al. 1999; Eiroa et al. 2001). This could indicate variability of the circumstellar disk around these objects, rather than the variable circumstellar obscuration 
commonly associated with large-amplitude optical brightness variations. Consequently these stars are good candidates for further studies.

The data presented here are suitable to be combined with the photometric data of the studied stars already present in the literature to perform a more detailed analysis of the photometric behaviour of the stars in our sample. However, the main value of the catalogue presented here may be for historical reference. It is well known that some young stars with well-documented historical variability (e.g. AB Aur, BN Ori, V351 Ori) have in recent years displayed a constant brightness for decades or longer. It is likely that some stars in our sample which have remained relatively constant over the time-frame studied here will become more active in years to come and vice-versa, that our variable stars may at some time cease to vary. In those cases, the information presented in Tables 2-4 will be extremely valuable in exploring the presently unknown cause of such long-term changes in photometric behaviour of young stars.

Acknowledgements. The authors would like to thank the staff at ESO La Silla and at the SAAO for the excellent help during the various observing runs. We would also like to thank several persons who spent long nights observing some of the data listed in this paper, and were not mentioned before. In alphabetic order: S. van Amerongen, E. A. Bibo, H. Cuypers, A. Hollander, M. Janssens, Y. K. Ng, R. van Ojik, L. Remijn and J. M. Smit. We are also indebted to Dr. A. van Genderen for his many years of work into maintaining the Walraven photometry programme on the Dutch telescope at La Silla. The referee, C. Catala, provided many suggestions for improvements of the paper, for which we thank him. This research has made use of the Simbad data base, operated at CDS, Strasbourg, France.

\section{References}

Bibo, E. A., \& Thé, P. S. 1991, A\&AS, 89, 319

Bouchet, P., Monetti, A., Slezak, E., Le Bertre, T., \& Manfroid, J. 1989, A\&AS, 80, 379
Bouchet, P., Manfroid, J., \& Schmider, F. X. 1991, A\&AS, 91, 409

Brand, J., \& Wouterloot, J. G. A. 1988, A\&AS, 75, 117

Carter, B. S. 1990, MNRAS, 242, 1

Carter, B. S., \& Meadows, V. S. 1995, MNRAS, 276, 734

de Geus, E., de Zeeuw, P. T., \& Lub, J. 1989, A\&A, 216, 44

de Winter, D., \& van den Ancker, M. E. 1997, A\&AS, 121, 275

Eiroa, C., Garzón, F., Alberdi, A., et al. 2001, A\&A, 365, 110

ESA 1997, The Tycho Catalogue, ESA SP-1200

Gezari, D. Y., Pitts, P. S., \& Schmitz, M. 1999, Catalog of Infrared Observations, 5th ed. (NASA Reference Publ.), 1294

Graham, J. A. 1982, PASP, 94, 244

Gosset, E., Surdej, J., \& Swings, J. P. 1984, A\&AS, 55, 411

Herbst, W., Herbst, D. K., Grossman, E. J., \& Weinstein, D. 1994, AJ, 108, 1906

Herbst, W., \& Shevchenko, V. S. 1999, AJ, 118, 1043

Kholopov, P. N., Samus, N. N., Frolov, M. S., et al. 1998, General Catalogue of Variable Stars (Nauka, Moscow)

Kilkenny, D., Whittet, D. C. B., Davies, J. K., et al. 1985, SAAO Circ., 9, 55

Manfroid, J, Sterken, C., Bruch, A., et al. 1991, A\&AS, 87, 481

Manfroid, J, Sterken, C., Cunow, B., et al. 1995, A\&AS, 109, 329

Shevchenko, V. S., Ezhkova, O., Tjin A Djie, H. R. E., et al. 1997, A\&AS, 124, 33

Shevchenko, V. S., Grankin, K. N., Ibragimov, M. A., Mel'Nikov, S. Y., \& Yakubov, S. D. 1993, Ap\&SS, 202, 121

Sterken, C., Jones, A., Vos, B., et al. 1996, IBVS, 4401, 1

Sterken, C., Manfroid, J., Anton, K., et al. 1993, A\&AS, 102, 79

Sterken, C., Manfroid, J., Beele, D., et al. 1995, A\&AS, 109, 329

Thé, P. S., de Winter, D., \& Pérez, M. R. 1994, A\&AS, 104, 351

van den Ancker, M. E., Thé, P. S., \& de Winter, D. 1996, A\&A, 309, 809

van den Ancker, M. E., de Winter, D., \& Tjin A Djie, H. R. E. 1998, A\&A, 330, 145 